\title{
Formação de Professores para o Uso Educacional de Tablets no Ensino Médio: possíveis mudanças na prática pedagógica
}

\section{Luciane M. Corte Real ${ }^{1}$, Mara Noble Rosane Tavares ${ }^{2}$, Jaqueline dos Santos Picetti ${ }^{3}$}

Faculdade de Educação - Universidade Federal do Rio Grande do Sul (UFRGS) ${ }^{1}$

Secretaria de Educação do Estado do Rio Grande do Sul (SEC) ${ }^{2}$

Secretaria do Município de Porto Alegre - $\mathrm{RS}^{3}$

luciane.real@ufrgs.br ${ }^{1}$; maratavares@professor.rs.gov.br² ; jaquelinepicetti@gmail.com³

Resumo: O presente relato é o recorte de uma pesquisa que está sendo realizada na Universidade Federal do Rio Grande do Sul. Apresenta-se as ações realizadas pelo Núcleo de Tecnologia em Educação do Rio Grande do Sul (NTE/RS) para distribuição e uso pedagógico dos Tablets pelos professores atuantes em sala de aula do Ensino Médio da Rede Pública Estadual. É realizado um mapeamento dos dados iniciais da pesquisa cuja questão principal é: quais são as possíveis mudanças pedagógicas provocadas pela inclusão das Tecnologias Móveis (Tablets) em sala de aula a partir da formação de professores? O objetivo é refletir sobre as referidas formações para implementação de ações de aprimoramento ao processo. Os dados iniciais apontam para a necessidade de construção de redes de aprendizagem a partir da formação pedagógica no NTE/RS.

Palavras-chave: Tablets, Formação de Professores, Práticas Pedagógicas.

Abstract: This report is part of a research that is being held in a Universidade Federal do Rio Grande do Sul. Presents the actions performed by the Center for Technology in Education do do Rio Grande do Sul (NTE / RS) for distribution and use of pedagogical Tablets by teachers working in the classroom of the School of Public Network State. It conducted a mapping of the initial data whose main question is: what are the possible pedagogical changes caused by the inclusion of Mobile Technologies (Tablets) in the classroom from the teacher training? The aim is to reflect on these formations to implement initiatives to improve the process. The initial data point to the need to build networks of learning from teacher training in NTE / RS.

Keywords: Tablets, Teacher Training, Pedagogical Practices.

\section{Apresentação}


Muitas pesquisas têm analisado a inclusão de Tablets na Educação Básica e Superior. Garcia (2010) investiga a visão de professores primários sobre a utilização de Tablets no ensino de Inglês. Oh e Gwizdka (2011) analisam a utilização de Tablets nas salas de aula do Ensino Superior, abordam como os estudantes usaram os Tablets para realizarem anotações, fazerem atividades em grupo e interagirem com o professor. Já Cantero et al (2013) pesquisam a utilização de Tablets para compreensão do espaço tridimensional.

O presente estudo trata de um recorte de uma pesquisa que iniciou em abril de dois mil e treze na Universidade Federal do Rio Grande do Sul e que se intitula "A Construção de Redes de Aprendizagem a Partir da Formação Pedagógica no Núcleo de Tecnologia em Educação (NTE) do Estado do RS". Nessa investigação o objetivo é analisar, a partir da formação pedagógica de professores no NTE/RS, as possíveis modificações em relação à prática pedagógica e a posição de professor e aluno na sala de aula e na escola. No presente artigo é apresentado as ações realizadas pelo Núcleo de Tecnologia em Educação do RS para distribuição e uso pedagógico dos Tablets pelos professores atuantes em sala de aula do Ensino Médio da Rede Pública Estadual, na zona urbana da cidade Porto Alegre.

Cabe destacar que um dos pressupostos da formação pedagógica do NTE/RS é a construção de uma educação cidadã. Esse núcleo de formação considera que não basta instalar Laboratórios de Informática (LI) nas escolas e utilizar o computador como substitutivo das ferramentas tradicionais usadas pelo professor em sala de aula, é necessário desenvolver um espaço e um tempo para a aplicação de arquiteturas pedagógicas. O LI e a sala de aula precisam ser espaços interdisciplinares, onde o professor atue como um desafiador de seus alunos, administrando o hardware, disponibilizando multimídias e recursos hipermidiáticos para um trabalho em parceria com seus colegas nos diferentes campos do saber.

\section{A pesquisa}

A relevância do tema pode ser construída com base na busca dos estudos recentes que foram desenvolvidos a respeito do uso das tecnologias móveis na escola.

Para Melhuish e Falloon (2010) apud Cantero et al (2013) o uso das tecnologias móveis nas escolas está redefinindo o espaço de aprendizagem. As tecnologias móveis nos possibilitam o rompimento do tempo e do espaço, assim como se baseiam em conectar pessoas e informações em conjunto através de ambientes de colaboração e comunidades virtuais. Heinrich (2012), investigou o uso do iPad como ferramenta para educação. Demonstrou um impacto significativo e muito positivo na aprendizagem dos estudantes, assim como mudanças a nível de desenvolvimento e pedagógicas. Esse pesquisador verificou que os alunos demonstraram grande aceitação dos dispositivos e um impacto significativo sobre a sua motivação, a sua capacidade de pesquisar, bem como de comunicação e colaboração. Também constatou que os professores estão aproveitando cada vez mais a variedade de aplicativos educacionais disponíveis.

Os pesquisadores Oh e Gwizdka (2011) relatam que várias investigações (Siegle e Foster, 2001; Saunders e Klemming, 2003; McVay et al, 2005;. Stephen, 2005;. Barak et al, 2006) destacam os efeitos positivos do uso do computador na educação, aumentando a 
participação e aprendizagem ativa, possibilitando melhores interações entre alunos e professores e aumentando a motivação e o desempenho acadêmico.

A investigação aqui apresentada está em fase inicial de desenvolvimento tendo como foco principal o seguinte problema:

Quais as possíveis mudanças pedagógicas provocadas pela inclusão das Tecnologias Móveis (Tablets) em sala de aula a partir da formação de professores para este fim?

O objetivo é de analisar, a partir da formação pedagógica de professores no NTE/RS, as possíveis modificações em relação à prática pedagógica e a posição de professor e aluno na sala de aula e na escola.

Trata-se de uma pesquisa qualitativa na forma de Estudo de Caso que visa aprofundar um tema (Yin, 2010). O Estudo de Caso é utilizado para a construção de uma investigação empírica que pesquisa fenômenos dentro de seu contexto real (Martins, 2008).

O estudo está sendo realizado a partir do NTE/RS. Os sujeitos são professores da Rede Estadual de Ensino, atuantes em sala de aula e que participam da formação para utilização dos Tablets Educacionais. Fazem parte da amostra os professores que aceitaram o convite para a participação e assinam o Termo de Consentimento Livre e Esclarecido.

Considera-se importante investigar esse espaço educativo, pois como diz Diaz (2012) a introdução das tecnologias na educação necessita de uma ação detalhada e programada de formação dos educadores. Para esse pesquisador as tecnologias possuem, por si mesmas, uma função educativa, mas que necessitam de uma formação de qualidade dos professores, para que sejam melhor exploradas.

A investigação divide-se em duas fases que estão acontecendo concomitantemente:

1. Estado da arte - levantamento de outras pesquisas que estão acontecendo no Brasil e que envolvam Escolas de Ensino Fundamental e Médio (pesquisas e relatos de experiências entre os anos de 2010 a 2013) e investigações internacionais. São utilizados pesquisadores que investigam o uso de diferentes recursos tecnológicos aliados a metodologias inovadoras como prática educativa proposta dentro dos Laboratórios de Informática das escolas para a construção de aprendizagens: Mattos et al, 2005; Haetinger et al, 2006; Coutinho, 2007; Real, Axt e Maraschin, 2007; Schäfer et al 2009; Gomes et al 2009; Nardin, Fruet e Bastos, 2009; Bittencourt et al, 2011; Real e Picetti, 2011; Real e Corbellini, 2011; Real e Tavares, 2012 além da experiência do grupo de pesquisa.

2. Acompanhamento das formações realizadas no NTE/RS e das propostas de professores utilizando os Tablets educacionais nas escolas.

Como instrumentos de pesquisa são utilizados questionários on-line, diário de campo da professora formadora e, quando necessário, entrevistas e observações nas escolas. Outros instrumentos serão construídos no próprio percurso da pesquisa.

O presente relato utiliza os diários de campo da professora formadora e de dois bolsistas que observaram algumas formações, bem como uma enquete realizada com um grupo de professores. 


\section{Relato de experiência}

As ações aqui relatadas foram realizadas pela Coordenadoria Regional de Educação do NTE/RS. O NTE/RS que é responsável por tornar possível a distribuição e o uso pedagógico dos Tablets Positivo YPY AB10DC, dez polegadas. Esses Tablets foram entregues para todos os professores atuantes em sala de aula no Ensino Médio da zona urbana, no período de abril a julho de 2013.

O uso dos Tablets no ensino público é uma ação do Proinfo Integrado, que é um programa do Ministério da Educação e Cultura (MEC). O Programa tem a finalidade propiciar a vivência de professores e alunos com a linguagem da tecnologia digital, garantindo tecnologia acessível com conectividade de internet. A introdução do Tablets visa oferecer aos professores um suporte no trabalho pedagógico e uma maior oportunidade de apropriação da cultura digital, suas linguagens e raciocínios. Os Tablets, atualmente, são um dos principais instrumentos de modernização tecnológica da Rede Estadual de Ensino.

Durante o período de abril a julho de 2013 foram atendidos setecentos e noventa e cinco dos mil e trinta e um professores de Ensino Médio. Esses professores foram convocados para a primeira etapa de distribuição dos dispositivos e formação para seu uso em sala de aula. Desse número, sete professores se recusaram a receber o Tablet, pois, entre as principais razões apresentadas, alegaram: "não ver aplicação pedagógica no dispositivo"; "não saber usar e não gostar de tecnologia" e, "pelo uso de tecnologia móvel ir contra a lei, que proíbe o uso de dispositivos móveis em sala de aula". Onze professores apresentaram as seguintes justificativas: estar em processo de Aposentadoria, estar em Licença Prêmio, estar em Licença Interesse, estar em Licença Saúde ou haver se Exonerado. Seis professores realizaram apenas um dos módulos, não apresentando disponibilidade para o outro. Duzentos e doze professores não atenderem à convocação feita pela SEC/RS, bem como não apresentaram justificativa.

O Plano de Formação foi dividido em dois módulos de quatro horas cada um, podendo ser realizado num dia inteiro ou em duas noites, conforme a realidade de cada escola atendida. O primeiro módulo apresenta as características, recursos e informações gerais, ensina a realizar o desbloqueio do usuário junto ao FNDE, fazer upgrade dos aplicativos e a explorar o dispositivo para familiarização com o uso da ferramenta e suas funcionalidades.

O formulário de avaliação do primeiro módulo contém dezesseis questões. Responderam à avaliação cento e dezesseis professores de trinta e nove escolas. Desses, noventa e cinco professores foram atendidos no NTE/RS e vinte e um receberam formação em suas próprias escolas. As questões de 1 a 3 referem-se aos dados de identificação. $\mathrm{O}$ formato das respostas às questões de número 4 a 15 ofereciam escolhas de 1 a 5 , onde 1 demonstrava fraca aderência ou intensidade e 5 forte aderência ou intensidade.

A seguir, é apresentado alguns dados do levantamento das questões de 4 a 15:

Os objetivos da Formação/Módulo foram atingidos?

\begin{tabular}{|l|l|}
1 & $1 \%$ \\
\hline
\end{tabular}


II Congresso Brasileiro de Informática na Educação (CBIE 2013)

Workshops (WCBIE 2013)

\begin{tabular}{|r|r|}
\hline 2 & $2 \%$ \\
\hline 3 & 16 \\
& $\%$ \\
\hline \multirow{2}{*}{4} & 34 \\
& $\%$ \\
\hline 5 & 47 \\
& $\%$ \\
\hline
\end{tabular}

A duração da Formação foi:

\begin{tabular}{|r|r|}
\hline 1 & $5 \%$ \\
\hline 2 & $11 \%$ \\
\hline 3 & $47 \%$ \\
\hline 4 & $21 \%$ \\
\hline 5 & $16 \%$ \\
\hline
\end{tabular}

Em nível Pedagógico os formadores foram:

\begin{tabular}{|r|l|}
\hline 1 & $2 \%$ \\
\hline 2 & $2 \%$ \\
\hline 3 & $13 \%$ \\
\hline 4 & $27 \%$ \\
\hline 5 & $57 \%$ \\
\hline
\end{tabular}

Os Equipamentos Pedagógicos disponibilizados foram utilizados:

\begin{tabular}{|r|r|}
\hline 1 & $0 \%$ \\
\hline 2 & $3 \%$ \\
\hline 3 & $8 \%$ \\
\hline 4 & $25 \%$ \\
\hline 5 & $65 \%$ \\
\hline
\end{tabular}

Os conteúdos abordados foram interessantes e pertinentes:

\begin{tabular}{|r|r|}
\hline 1 & $2 \%$ \\
\hline 2 & $4 \%$ \\
\hline 3 & $12 \%$ \\
\hline 4 & $36 \%$ \\
\hline 5 & $47 \%$ \\
\hline
\end{tabular}

O nível de tratamento dos assuntos foi:

\begin{tabular}{|r|l|}
\hline 1 & $6 \%$ \\
\hline 2 & $3 \%$ \\
\hline 3 & $11 \%$ \\
\hline 4 & $44 \%$ \\
\hline 5 & $36 \%$ \\
\hline
\end{tabular}

As ações/dinâmicas realizadas durante a Formação foram: 


\begin{tabular}{|r|l|}
\hline 1 & $3 \%$ \\
\hline 2 & $3 \%$ \\
\hline 3 & $14 \%$ \\
\hline 4 & $41 \%$ \\
\hline 5 & $41 \%$ \\
\hline
\end{tabular}

O Material de Apoio entregue foi:

\begin{tabular}{|r|r|}
\hline 1 & $7 \%$ \\
\hline 2 & $5 \%$ \\
\hline 3 & $15 \%$ \\
\hline 4 & $27 \%$ \\
\hline 5 & $46 \%$ \\
\hline
\end{tabular}

Estou utilizando o Tablet no desempenho de minha profissão:

\begin{tabular}{|r|r|}
\hline 1 & $8 \%$ \\
\hline 2 & $7 \%$ \\
\hline 3 & $32 \%$ \\
\hline 4 & $34 \%$ \\
\hline 5 & $19 \%$ \\
\hline
\end{tabular}

A questão 16 ofereceu aos professores um campo opcional de opinião ou sugestões para as próximas formações. Algumas das respostas:

"dividir o grupo entre aqueles que já acessam e utilizam com facilidade e os que necessitam de informações mais básicas", "as programações poderiam ser desenvolvidas por assuntos mais específicos...", "Mais da metade do tempo da formação fiquei sem ter como avançar por que os temas abordados e as especificidades apresentadas já fazem parte daquilo que utilizo a pelo menos dois anos"

Com base nas respostas pode-se verificar a necessidade de aprofundamento da temática, visto que os conhecimentos básicos já eram de conhecimento de muitos dos professores.

O segundo módulo ofereceu aos professores uma formação sobre como usar o Tablet como ferramenta pedagógica, assim como a apresentação dos recursos contidos no dispositivo que podem ser incorporados na prática de sala de aula.

No questionário de opinião, do segundo módulo, foram avaliadas quarenta e oito respostas de professores de dezenove escolas. As questões iniciais envolvem a identificação. As quatro questões seguintes solicitam respostas descritivas sobre a utilização de tecnologia digital nas aulas. No quadro abaixo é apresentada algumas respostas dos professores (são mantidas as escritas dos professores):

-Acho que os professores tem que se preparar muito ainda.

-Pode agilizar a pesquisa dos alunos em sala de aula, bem como a facilidade de 
baixar livros de literatura.

-Acredito que é um recurso mais acessível e prático para utilização em sala de aula.

-Através de atividades de pesquisa, tarefas via email e apresentação de trabalhos.

-Como apoio didático.

-Auxiliando nas aulas de produção textual e língua portuguesa.

-Acredito que nossas aulas terão mais interatividade.

-Através de aplicativos instalados no Tablet, poderei desenvolver alguns conteúdos como geometria.

-Se pensarmos em motivação, estaremos marcando muitos pontos. Eles apresentarão mais entusiasmo em poder interagir de maneira tão lúdica e rápida. -Alguns alunos já utilizam este recurso em sala de aula, porém tem que existir um planejamento bem estruturado para que não se utilize este recurso apenas para acessar redes sociais.

-A reação dos alunos foi super natural. Ninguém se surpreendeu. Eles estão acostumados com a tecnologia, e nós professores já as utilizamos na escola a muito tempo, com os computadores da própria escola, os data-shows, que permitem de forma muito mais acessível mostra algo, trabalhar algum vídeo, mostrar algum simulador, na internet, e para todos os alunos ao mesmo tempo. -Acho que se surpreenderão com curiosidade e questionamentos. Acho que gostarão.

-Creio que eles irão participar mais nas aulas. Acredito que para a maioria não dará maior importância...

-No início vão se dispersar e criticar o professor por ele poder usar Tablet e eles não poderem utilizar o telefone celular ...mas depois, vendo que há vantagens para eles, acho que vamos otimizar tempo e outros recursos. é mais uma maneira de chamar a atenção dos alunos Os alunos se sentem muito estimulados com as ferramentas digitais, surpresos por as tecnologias estarem sendo utilizadas na rede estadual, no entanto cobrando que o Tablet seja mais e melhor utilizado.

-Ficaram felizes, por estarmos integrados a revolução tecnológica.

-Acredito que eles tendem a participar mais das aulas, mas isso já faz parte da constituição dos jovens de hoje. Esse tipo de tecnologia é muito mais interessante para nós. Embora não seja algo legal de estar dizendo, é inevitável, tenho que cuidar do Tablet para que os alunos não o roube. Há na escola casos semanais de roubo de celulares. Isso é um fator preocupante.

Com base nas respostas dos professores, preliminarmente, pode-se verificar o quanto o uso dos Tablets poderão ressignificar o trabalho pedagógico em sala de aula.

\section{Considerações finais}


Nesse artigo é apresentado alguns dados de uma pesquisa inicial, mas pode-se observar que as formações são um fator provocativo para muitos professores, levando-os a desejarem aprender a aprender como usar o Tablet em sala de aula com seus alunos.

Muitos professores que participaram das formações retornam ao NTE/RS solicitando aprofundamento dos conhecimentos necessários para o uso dos Tablets.

Vários professores deram retorno de como tem ocorrido o trabalho em sala de aula. Eles enviam fotos e relatos sobre suas práticas com a utilização do Tablet através de e-mail, telefonemas e pessoalmente, demonstrando estarem desenvolvendo atividades e projetos com seus alunos.

Contudo, o território das formações não tem se constituído um espaço tranquilo. No transcorrer das formações, vários professores apresentaram sentimentos contraditórios: queixas em torno das políticas públicas na Educação e o tipo de investimentos aplicados; em relação as suas reais capacidades para utilizar a tecnologia com os alunos no processo de aprendizagem; a dificuldade em envolver os alunos nos conteúdos; a superação do domínio das redes sociais e jogos pedagógicos durante as aulas; entre outras questões.

A tarefa de formar professores para utilizar as Tecnologias da Informação e da Comunicação, muitas vezes, não consegue preparar esses profissionais para o desafio que seu uso, em sala de aula, representa. A formação continuada dos professores para o uso das tecnologias está comprometida em evidenciar a necessidade de mudar o foco do processo do ensino para a aprendizagem, mostrando que professores e alunos não são agentes antagônicos no processo, mas parceiros na busca de soluções e construção de conhecimentos.

Com base nos dados iniciais da pesquisa, verifica-se a necessidade de construção de redes de aprendizagem a partir da formação pedagógica no NTE/RS, para atender as necessidades que o uso dos Tablets em sala de aula geram.

\section{Referências Bibliográficas}

Bittencourt, Juliano; Kist, Sílvia; Tatizana, Décio; Schafer, Patrícia; Fagundes, Léa (2011) Ambientes virtuais de aprendizagem na modalidade 1:1: um estudo de caso sobre o AMADIS na Fase I do Programa UCA. . RENOTE - Revista Novas Tecnologias na Educação, Porto Alegre, v. 9, n. 2.

Cantero, Jorge De La Torre; Martin-Dorta, Pérez; Carrera, Carlos Carbonell; González, Manuel Contero (2013). Entorno de aprendizaje ubicuo con realidad aumentada y tabletas para estimular la comprensión del espacio tridimensional. RED. Revista de Educación a Distancia. Número 37 Año XII. Número 37. 15 de Abril de 2013, España.

Coutinho, Clara Pereira (2007). Tecnologia Educativa e Currículo: caminhos que se cruzam ou se bifurcam? Em: 
http://www.periodicos.proped.pro.br/index.php?journal=revistateias\&page=article\&op= view\&path $\% 5 \mathrm{~B} \% 5 \mathrm{D}=176>$ acesso em 28 de setembro de 2013.

Diaz, Tello J. (2012). Investigación y aprendizaje en las aulas a través de la integración de las tecnologías de la información y la comunicación. Aularia, 1(1) Enero. pp: 17-22, janeiro de 2012.

Garcia, Maria Luisa. Professorado de Primaria y aplicación de tecnologias: um estúdio de caso. Universidad Nacional de Educación a distancia (2010). Pixel-Bit. Revista de Medios y Educación. N 38 Julio- Diciembre 2010 pp. 63 - 7, España.

Gomes, Mayra; Soares, Rosana; Leite, Andrea. Wiki: uma experiência pedagógica. Revista Online de Comunicação Linguagem e Mídias (2009). Em: www.rumores.usp.br/gomes.pdf acesso em 28 de setembro de 2013.

Haetinger, Daniela; Rela, Eliana; Gelatti, Lilian; Konrath, Mary Lúcia; Kist, Tânia; Carvalho, Marie Jane; Nevado, Rosane (2006). Formação de Professores e Práticas Pedagógicas no Contexto Escolar das Séries Iniciais. RENOTE - Revista Novas Tecnologias na Educação, Porto Alegre, v. 4, n. 2.

Lei de Diretrizes e Bases da Educação Brasileira (1996). LEI No 9.394 de 20 de Dezembro de 1996. Em: < http://portal.mec.gov.br/seed/arquivos/pdf/tvescola/ Leis/lein9394.pdf>

acesso em 28 de setembro de 2013.

Lei de Diretrizes e Bases da Educação Brasileira (2005). DECRETO N 5.622, DE 19 DE DEZEMBRO DE 2005. Em: < http://www.planalto.gov.br/ccivil 03/_Ato20042006/2005/ decreto/D5622.htm> acesso em 28 de setembro de 2013.

Mattos, Eduardo Britto; Júnior, José Carlos; Mattos, Milena Vitello (2005) Projetos de Aprendizagem e o Uso de TIC's - Tecnologias de Informação e Comunicação: Novos Possíveis na Escola. RENOTE - Revista Novas Tecnologias na Educação, Porto Alegre, v. 3, n. 2.

Nardin, A.C.; Fruet, F.S.O. ; Bastos (2009) Potencialidades Tecnológicas e Educacionais em Ambiente Virtual de Ensino-Aprendizagem. RENOTE, Revista Novas Tecnologia na Educação, V. 7 n. 3.

Oh, Kyong eun e Gwizdka, Jacek (2011) Impatient opportunists: a study of technology use in a higher education classroom. Journal of Applied Research in Higher Education Vol. 3 No. 2, 2011 pp. 81-96.

Picetti, J. S. e Real, L. M. C.; (2011). Aprendizagens por possibilidades de deslocamentos em um Laboratório de Informática: um estudo de caso no Ensino Fundamental. In: $22^{\circ}$ Simpósio Brasileiro de Informática na Educação, 2011, Aracaju. Anais do XXII SBIE XVII WIE, v. Trilha. p. 1-4. 
Real, L. M. C. ;Axt, Margarete ; Maraschin, Cleci (2007) Projetos de Aprendizagem e Tecnologias Digitais: uma experiência promovendo transformações na convivência na escola. RENOTE. Revista Novas Tecnologias na Educação, v. 5, p. 9c.

Real, L. M. C.; Corbellini S. (2011). Proposta de uso de Wiki como Arquitetura Pedagógica: cooperação. In: XXII Simpósio Brasileiro de Informática na Educação e XVII Workshop de Informática na Escola (SBIE e WIE).

Real, L. M. C.; Tavares, M. R. N. (2012). Alunos do Ensino Fundamental e professores construindo espaços de Educação a Distância na Escola. In: 23 Simpósio Brasileiro de Informática na Educação, Congresso Brasileiro de Informática na Educação, 2012, Rio de Janeiro. Anais do XXIII CBIE - XVIII WIE 2012 em: < http://www.brie.org/pub/index. php/wie/article/view/2086>

RESOLUÇÃO N¹96/96 versão 2012. MINISTÉRIO DA SAÚDE CONSELHO NACIONAL DE SAÚDE COMISSÃO NACIONAL DE ÉTICA EM PESQUISA em: $<$ http://conselho.saude.gov.br/web_comissoes/conep/aquivos/resolucoes/23_out _versao_final_196_ENCEP2012.pdf $>$

Schäfer, Lacerda e Fagundes, (2009). Escrita colaborativa na cultura digital: ferramentas e possibilidades de construção do conhecimento em rede RENOTE. Revista Novas Tecnologias na Educação, v. 7, n. 1. 